\title{
miR-24-3p regulates bladder cancer cell proliferation, migration, invasion and autophagy by targeting DEDD
}

\author{
GUOQIANG YU, ZHAOHUI JIA and ZHONGLING DOU \\ Department of Urological Surgery, The First Affiliated Hospital and College of Clinical Medicine \\ of Henan University of Science and Technology, Luoyang, Henan 471003, P.R. China
}

Received May 22, 2016; Accepted November 14, 2016

DOI: $10.3892 / o r .2016 .5326$

\begin{abstract}
RNAs (miRNAs), a class of small non-coding RNA molecules, can regulate gene expression by interacting with the 3'-untranslated regions (3'UTR) of target genes and influence various biological processes. We investigated the potential role of miR-24-3p in the development of bladder cancer by regulating DEDD, a member of the death effector domaincontaining protein family. First, we found that miR-24-3p was highly expressed and that DEDD was expressed at a low level in bladder cancer tissues compared with that in adjacent bladder tissues by qRT-PCR $(\mathrm{P}<0.0001)$. Second, we found that miR24-3p promoted the proliferation ability of bladder cancer cells using the MTT assay and colony forming assay; and showed that miR-24-3p accelerated the migration and invasion of bladder cancer cells using migration and invasion assays $(\mathrm{P}<0.05)$. Moreover, miR-24-3p inhibited apoptosis of bladder cancer cells, as shown by flow cytometry $(\mathrm{P}<0.05)$. Western blot results demonstrated that miR-24-3p participated in autophagy of bladder cancer cells by DEDD. In addition, the tumor formation assay showed that miR-24-3p promoted the growth of bladder tumor in vivo. Furthermore, the luciferase reporter gene assay indicated that miR-24-3p suppressed DEDD gene transcription. Therefore, our study indicated that miR-24-3p promoted bladder cancer progression by inhibiting DEDD.
\end{abstract}

\section{Introduction}

Human bladder cancer is prevalent throughout the world. According to statistics, human bladder cancer is the fourth leading cause of malignancy in men and currently the tenth most malignant tumor in women (1). According to its pathological features, bladder cancer can be divided into two major

Correspondence to: Dr Zhongling Dou, Department of Urological Surgery, The First Affiliated Hospital and College of Clinical Medicine of Henan University of Science and Technology, 24 Jinghua Road, Jianxi, Luoyang, Henan 471003, P.R. China

E-mail: douzhongling2016@sina.com

Key words: miR-24-3p, DEDD, bladder cancer, migration and invasion groups: urothelial carcinoma (UC) and invasive bladder cancer. $\mathrm{UC}$ is the most widespread bladder cancer, and it frequently recurs, but rarely progresses $(2,3)$; invasive bladder cancer easily progresses to distant metastases (4). However, because the pathophysiological mechanisms underlying bladder cancer are not clear, the appropriate treatment to increase bladder cancer survival rates is limited. Consequently, molecular mechanism studies concerning the development mechanisms of bladder cancer are urgent.

microRNAs (miRNAs) are a subgroup of endogenous non-coding small RNAs that are 19-23 nucleotides in length and are involved in post-transcriptional regulation by targeting the 3'UTR of mRNA to affect biological processes (5-8). An increasing number of studies have shown that miRNAs act as a group of regulatory genes and participate in the development and progression of various diseases, such as the progression of tumorigenesis (6,9-11). Many studies have revealed that various miRNAs, such as miR-126, miR-143, miR-145, miR-23b, and miR-144-5p, are related to the development of bladder cancer (12-16). The mechanism and function of miR24-3p have also been studied in glioma (17), lung cancer (18), hepatocellular carcinoma (19), colorectal cancer (20), and breast cancer (21). However, the functional relevance of miR24-3p in bladder cancer is not fully understood.

DEDD, a member of the death effector domain-containing protein family, participates in biological processes, such as cell apoptosis, cell cycle, and cell mitosis $(22,23)$. Increasing evidence has shown that the epithelial-mesenchymal transition (EMT), a crucial development process, promotes cancer invasion and metastasis $(24,25)$. Studies have found that DEDD serves as a novel tumor repressor and can suppress EMT and metastasis in breast and colon cancers $(26,27)$. Therefore, DEDD may act as a prognostic marker and potential therapeutic target for the treatment of cancer metastasis. However, the mechanism and function of DEDD have not been reported in bladder cancer. To further study the anti-metastatic roles of DEDD in bladder cancer, our study investigate the expression level of DEDD in bladder cancer tissues and the interaction relationship between DEDD and miR-24-3p in bladder cancer.

Here, we confirmed that miR-24-3p was highly expressed and that DEDD was expressed at a low level in bladder cancer tissues. miR-24-3p promoted the abilities of proliferation, migration and invasion; inhibited apoptosis; participated in autophagy of bladder cancer cells by LC3, DEDD, and p62 
in vitro; and promoted the growth of bladder tumor in vivo. Furthermore, miR-24-3p suppressed DEDD gene transcription and accelerated proliferation and invasion through DEDD in bladder cancer cells. Therefore, miR-24-3p could be a pivotal potential therapeutic target for the treatment of bladder cancer.

\section{Materials and methods}

Cell lines and transfection. Human ureter epithelium (HCV29) cells, the human bladder cancer cell line T24, human bladder transitional cell carcinoma (UM-UC-3) cells, human bladder cancer (HBC) cells, and malignant bladder carcinoma (BLU87) cells were purchased from College of Life Science, Hunan Normal University, China. All of the cells were cultured in Dulbecco's modified Eagle's medium (DMEM; Gibco-Invitrogen, Carlsbad, CA, USA) containing 10\% fetal bovine serum (FBS; Invitrogen, Carlsbad, CA, USA), penicillin (100 U/ml; Invitrogen), and streptomycin (100 $\mu \mathrm{g} /$ $\mathrm{ml}$; Invitrogen). All of the cultures were maintained at $37^{\circ} \mathrm{C}$ with $5 \% \mathrm{CO}_{2}$. For treatment, according to the manufacturer's protocol, T24 and HBC cells $\left(2 \times 10^{5}\right.$ cells/well) were seeded in 6-well plates and were transfected with $200 \mu 1$ of mature miR-24-3p mock, mimic, scramble, or inhibitor (GenePharma Co., Ltd., Shanghai, China) using Lipofectamine ${ }^{\mathrm{TM}} 3000$ (Invitrogen, Carlsbad, CA, USA) for $72 \mathrm{~h}$. Similarly, T24 and $\mathrm{HBC}$ cells were transfected with scramble and vector, miR-24-3p and pcMV6, and miR-24-3p and DEDD with Lipofectamine 3000 , respectively.

Clinical specimens. Our study obtained ethics committee approval from the First Affiliated Hospital of Henan University of Science and Technology. We collected the bladder cancer tissues and adjacent non-cancerous tissues samples from the First Affiliated Hospital of Henan University of Science and Technology between 2014 and 2016. All of the tissue samples were snap-frozen at $-80^{\circ} \mathrm{C}$. Each patient provided written informed consent.

Quantitative real-time reverse transcription PCR ( $q R T-P C R)$. According to the manufacturer's instructions, total RNA was extracted from bladder cancer tissues, matched adjacent noncancerous tissues and treated T24 and HBC cells using the RNeasy Plus Mini kit. cDNAs were synthesized using the RevertAid First Strand cDNA Synthesis kit (Thermo Fisher). The samples were normalized to U6 according to their miRNA and GAPDH for mRNA. As previously described (28), the mRNA expression levels were analyzed using the SYBR-Green PCR Master Mix kit (Takara). For the detection of mRNA, the primer sequences for GAPDH were: 5'-TGT TCG TCA TGG GTG TGA AC-3' (the forward primer) and 5'-ATG GCA TGG ACT GTG GTC AT-3' (the reverse primer); the primer sequences for DEDD were: 5'-TCC CCA GCC CTC TAA AAC AG-3' (the forward primer) and 5'-CCG CAG TCT GAT GTC ACA TG-3' (the reverse primer); the primer sequences for PAK1 were: 5'- TTG GGA ATG GAT GGC TCT GT-3' (the forward primer) and 5'-ATG TCA ACC TTG GGC CCA TA-3' (the reverse primer). For the detection of miRNA, the primer sequence for miR-24-3p was 5'-CCC ATT CAG CAG GAA CAG AAA-3' and that for U6 was 5'-ACG CAA ATT CGT GAA GCG TT-3'.
Western blot analysis. Treated T24 and HBC cells were lysed using RIPA buffer containing a protease inhibitor cocktail (P8340; Sigma). Equivalent protein samples were separated on $8 \%$ SDS/PAGE gels and were then transferred to PVDF membranes (Millipore, Billerica, MA, USA). The PVDF membranes were incubated in 5\% skim milk (BD Biosciences) for $2 \mathrm{~h}$ with a primary antibody at $4^{\circ} \mathrm{C}$ overnight, followed by incubation with horseradish peroxidase-conjugated secondary antibodies for $1 \mathrm{~h}$ at room temperature. The results were obtained using an enhanced chemiluminescence detection system (Amersham Biosciences, Piscataway, NJ, USA). In this study, the used primary antibodies were anti-LC3 (1:200; MBL International Co., Woburn, MA, USA), anti-DEDD (1:200; ab56480, Abcam), and anti-P62 (1:5000, Progen, GP62-C); the anti-GAPDH antibody (1:4000, Beverly, MA, USA) was used as an internal control.

Luciferase reporter assays. Treated $\mathrm{T} 24$ and $\mathrm{HBC}$ cells $\left(5 \times 10^{4}\right.$ cells/well) were cultured in 24 -well plates and were co-transfected with miR-24-3p and the wild-type pGL3-promoter-DEDD reporter vector or mutant pGL3promoter-DEDD reporter vector as well as a renilla plasmid (RL-SV40) using Lipofectamine 3000 (Invitrogen). The renilla plasmid was used as an internal control. According to the manufacturer's instructions, the luciferase activities were measured by the Dual-Luciferase reporter assay system (Promega, Madison, WI, USA).

Colony forming unit (CFU) assay. For the colony forming unit assay, T24 and HBC cells $\left(5 \times 10^{3}\right.$ cells/well) transfected with miR-24-3p mock, mimics, scramble, or inhibitors were cultured into a $10-\mathrm{cm}$ culture dish with complete DMEM medium for 12 days. Next, the colonies were fixed using methanol and dyed using Giemsa dye solution. The colony forming units were observed and photographed.

MTT assay. T24 and HBC cells ( $3 \times 10^{3}$ cells/well) transfected with miR-24-3p mock, mimics, scramble, or inhibitors were seeded in 96-well plates for $0,12,24,48$, and $72 \mathrm{~h}$. Next, $20 \mu \mathrm{l}$ of a 3-(4,5-dimethylthiazol-2-yl)-2,5-diphenyltetrazolium bromide (MTT, $5 \mathrm{mg} / \mathrm{ml}$ ) solution was added into each well. After $4 \mathrm{~h}$, formazan was dissolved in a dimethyl sulfoxide (DMSO) solution. A microplate reader was used to measure the absorbance at $570 \mathrm{~nm}$.

Flow cytometric analysis of the cell apoptosis. The treated T24 and HBC cells were resuspended in PBS and were stained with FITC-Annexin V and propidium iodide (PI). The flow cytometry results were analyzed using FlowJo software. The cells were segmented into four types, viable cells, dead cells, early-stage apoptotic cells, and late-stage apoptotic cells.

Migration and invasion assays. According to the manufacturer's instructions, the migration abilities of the treated T24 and HBC cells were carried out in 24-well Transwell chambers with $8-\mu \mathrm{m}$ pore cell culture inserts (Costar). The treated cells $\left(5 \times 10^{5}\right.$ cells/well) in serum-free media were seeded into the upper chamber, and DMEM medium with $10 \% \mathrm{FBS}$ was seeded into the lower chambers for $24 \mathrm{~h}$ in an incubator at $37^{\circ} \mathrm{C}$ with $5 \% \mathrm{CO}_{2}$. Thereafter, the migratory cells 
A

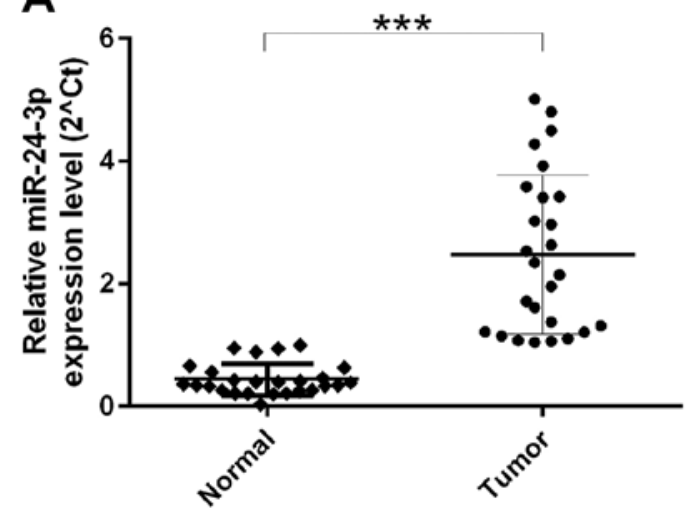

C

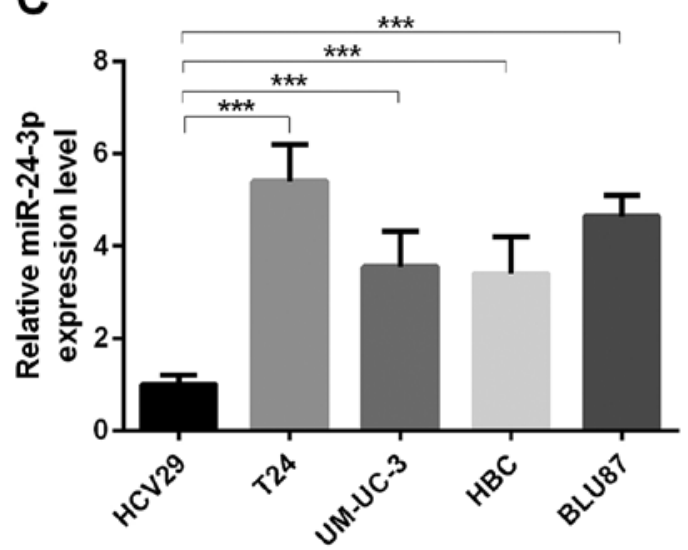

B
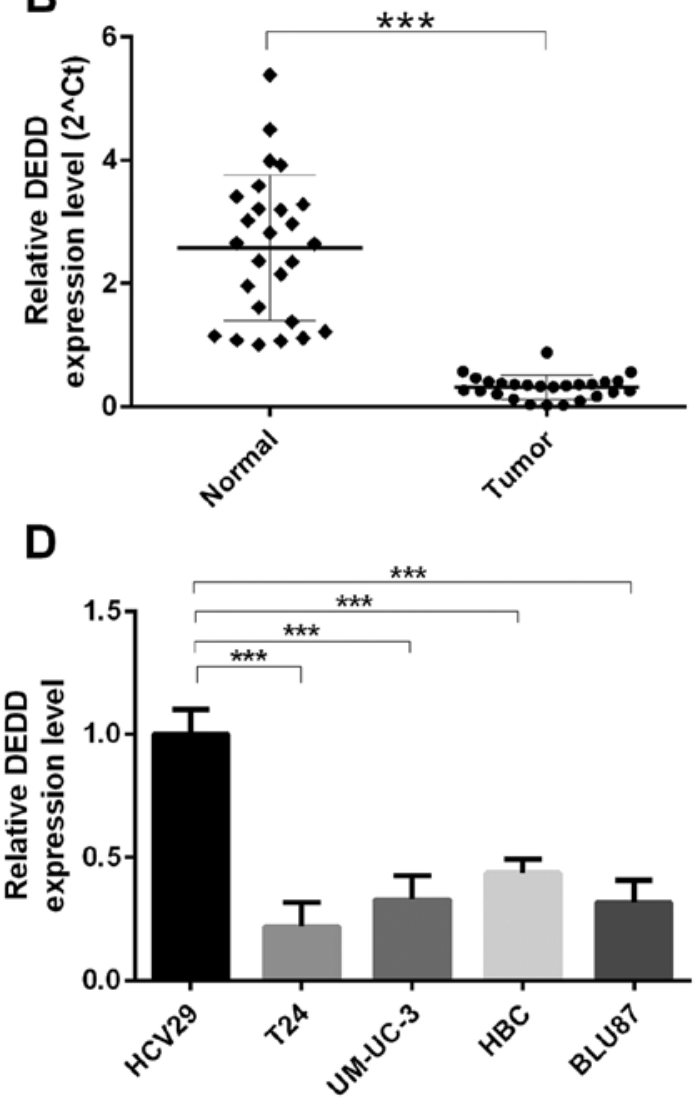

Figure 1. miR-24-3p overexpression and DEDD low-expression in bladder cancer. (A) miR-24-3p expression was analyzed by qRT-PCR in bladder cancer tissues $(n=26)$ and adjacent bladder tissues $(n=26)\left({ }^{* * *} \mathrm{P}<0.001\right)$. (B) qRT-PCR was used to detect the mRNA expression level of DEDD in bladder cancer tissues $(n=26)$ and adjacent bladder tissues $(n=26)\left({ }^{* * *} \mathrm{P}<0.001\right)$. (C) The mRNA expression level of miR-24-3p was detected by qRT-PCR in human ureter epithelium (HCV29) cells and bladder cancer cells (T24, UM-UC-3, HBC, and BLU87) $\left({ }^{* * *} \mathrm{P}<0.001\right)$. (D) The mRNA expression level of DEDD was detected by qRT-PCR in human ureter epithelium (HCV29) cells and bladder cancer cells $\left(\right.$ T24, UM-UC-3, HBC, and BLU87) $\left(^{* * *} \mathrm{P}<0.001\right)$.

were fixed, stained and counted under a microscope. For the invasion assay, $10 \mu 1$ of 1:5 diluted matrigel (BD Biosciences) was pre-paved into Transwell inserts before the experiment at $37^{\circ} \mathrm{C}$ for $2 \mathrm{~h}$.

Tumor formation in nude mice. The Institutional Committee for Animal Research approved the animal experiments of this study, and we performed tumor formation in nude mice according to the Institutional Animal Care and Use Committee. The flanks of 5-week-old BALB/c athymic nude mice were subcutaneously implanted with $\mathrm{T} 24$ cells $\left(1 \times 10^{7}\right.$ cells in $100 \mu \mathrm{l})$ transfected with miR-24-3p mimics, mock, inhibitor or scramble for $0,8,16,24,32,40$, and 48 days. The tumor volume and tumor weight were measured.

Statistical analysis. The data were analyzed by Student's t-test and analysis of variance (ANOVA) using Prism6 (GraphPad Software, Inc., San Diego, CA, USA) and SPSS 15.0 software (SPSS, Chicago, IL, USA). All of the results are presented as the means \pm SD. A P-value $<0.05$ was considered to indicate a statistically significant difference.

\section{Results}

miR-24-3p is overexpressed, and DEDD expression is low in bladder cancer. We collected bladder cancer tissues and paired non-carcinoma tissues from 26 patients. The mRNA expression levels of miR-24-3p and DEDD were measured by $\mathrm{qRT}$-PCR. The results indicated that the expression level of miR-24-3p was increased in bladder cancer tissues $(n=26)$ compared with that in para-carcinoma tissues $(n=26)$ $(\mathrm{P}<0.0001)$ (Fig. 1A); the mRNA expression level of DEDD was decreased in bladder cancer tissues $(n=26)$ compared with that in para-carcinoma tissues $(n=26)(P<0.0001)$ (Fig. 1B). We also found that the mRNA expression level of miR-24-3p was increased in human ureter epithelium (HCV29) cells compared with that in bladder cancer cells (T24, UM-UC-3, HBC, and BLU87) $(\mathrm{P}<0.0001)$. The highest level of miR-24-3p was found in T24 cells $(\mathrm{P}<0.0001)$ (Fig. 1C); the mRNA expression level of DEDD was decreased in human ureter epithelium (HCV29) cells compared with that in bladder cancer cells (T24, UM-UC-3, HBC, and BLU87) (P<0.0001). The lowest level of DEDD was found in T24 cells $(\mathrm{P}<0.0001)$ (Fig. 1D).

miR-24-3p promotes cell proliferation and invasion in bladder cancer cells. The impact of miR-24-3p on the proliferation and invasion abilities of bladder cancer cells was detected in T24 and HBC cells transfected with miR-24-3p mimics, mock (control), inhibitor or scramble (control). First, the mRNA expression level of miR-24-3p was measured by qRT-PCR. Our results indicated that the transfection effects of miR-24-3p mimics and inhibitor were high $(\mathrm{P}<0.05)$ (Fig. 2A). 

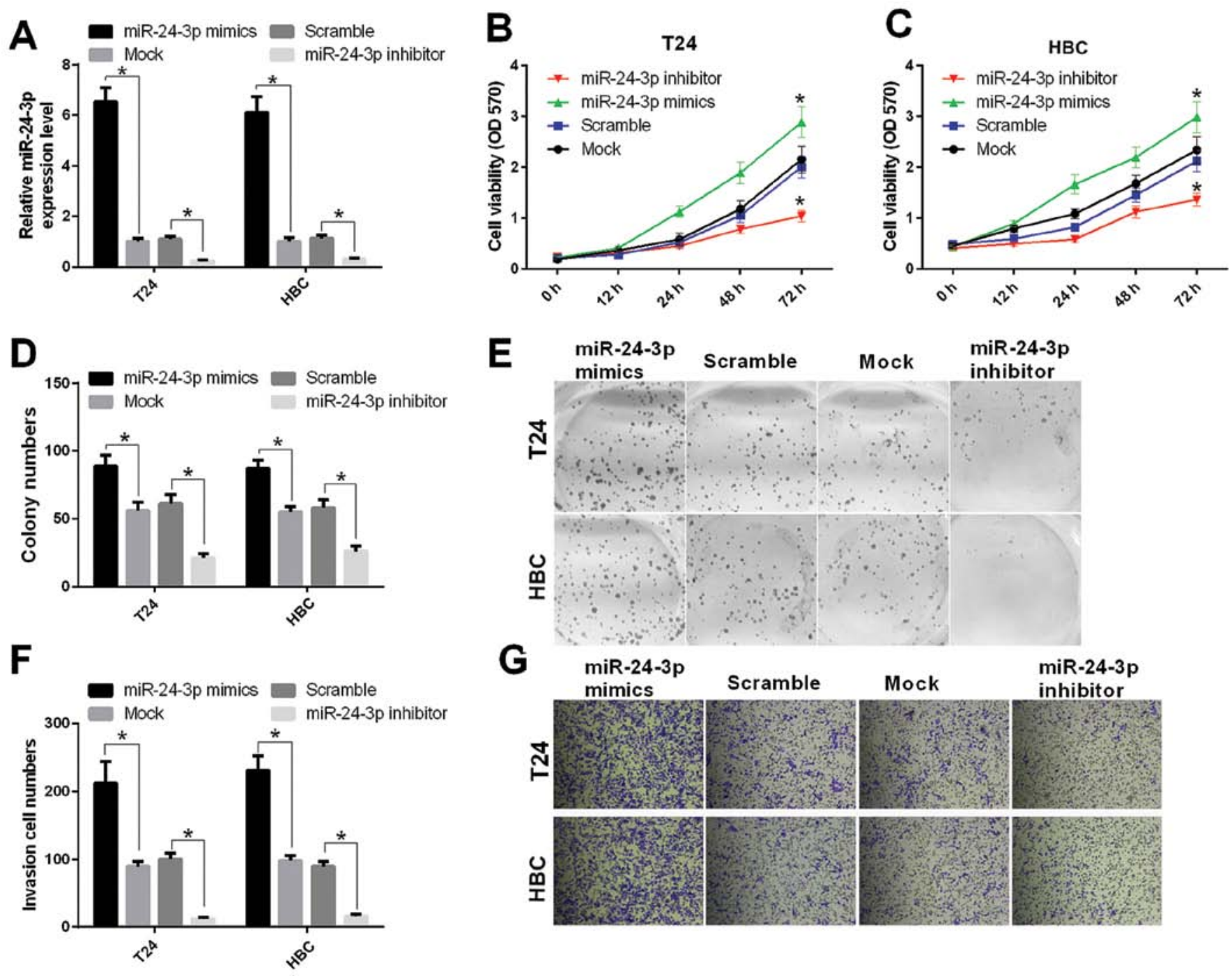

Figure 2. miR-24-3p promotes the abilities of cell proliferation and invasion in bladder cancer cells. (A) The mRNA expression level of miR-24-3p was measured by qRT-PCR in T24 and HBC cells transfected with miR-24-3p mimics, mock (control), inhibitor or scramble (control), respectively (*P<0.05). (B) The MTT assay was performed to detect proliferation of T24 cells transfected with miR-24-3p mimics, mock, inhibitor or scramble, respectively, at 0 , $12,24,48$, and $72 \mathrm{~h}\left({ }^{*} \mathrm{P}<0.05\right)$. (C) The MTT assay was used to measure the proliferation of Hbc cells transfected with miR-24-3p mimics, mock, inhibitor or scramble, respectively $\left({ }^{*} \mathrm{P}<0.05\right)$. (D) The colony numbers were counted $\left({ }^{*} \mathrm{P}<0.05\right)$. (E) Cell proliferation was detected by colony forming assay in $\mathrm{T} 24$ and $\mathrm{HBC}$ cells transfected with miR-24-3p mimics, mock, inhibitor or scramble. (F) The invasion cell numbers were counted ( $\mathrm{P}<0.05)$. (G) Cell invasion was determined by the Transwell assay in T24 and HBC cells transfected with miR-24-3p mimics, mock, inhibitor or scramble, respectively.

The bladder cell proliferation ability was detected by the MTT assay, and the results indicated that miR-24-3p promoted the proliferation of $\mathrm{T} 24$ cells $(\mathrm{P}<0.05)$ (Fig. 2B) and $\mathrm{HBC}$ cells $(\mathrm{P}<0.05)$ (Fig. 2C). Similarly, the colony forming unit assay was performed to determine the bladder cell proliferation. Our results found that the proliferation ability was significantly increased in T24 and HBC cells transfected with miR-24-3p mimics relative to mock and that the proliferation ability was significantly decreased in T24 and HBC cells transfected with miR-24-3p inhibitor relative to scramble $(\mathrm{P}<0.05)$ (Fig. 2D and $\mathrm{E})$. Furthermore, we found that miR-24-3p promoted the invasion ability of T24 and HBC cells (Fig. 2F and G).

miR-24-3p promotes cell migration, inhibits apoptosis, and participates in autophagy. We further studied the cell migration and apoptosis that were affected by miR-24-3p in bladder cancer cells. First, the migration ability of T24 and HBC cells transfected with miR-24-3p mimics, mock, inhibitor or scramble were measured by the migration assay. The results indicated that the migration of T24 and HBC cells transfected with miR-24-3p mimics were significantly increased compared with mock $(\mathrm{P}<0.05)$; the cell migration capacity of T24 and HBC cells transfected with the miR24-3p inhibitor was significantly decreased compared with scramble $(\mathrm{P}<0.05)$ (Fig. 3A and $\mathrm{B})$. Second, cell apoptosis was measured by Annexin V-FITC/PI staining. The results showed that apoptosis T24 and HBC cells transfected with miR-24-3p mimics was significantly decreased compared with that of the mock $(\mathrm{P}<0.05)$; apoptosis of T24 and HBC transfected with miR-24-3p inhibitor was significantly increased compared with that of scramble $(\mathrm{P}<0.05)$ (Fig. $3 \mathrm{C}$ and D). Furthermore, the western blot results showed that miR-24-3p upregulated the protein expression levels of LC3 and p62 and downregulated the protein expression level of DEDD in T24 and HBC cells (Fig. 3E). These results indicated that miR-24-3p participated in autophagy by repressing 
A
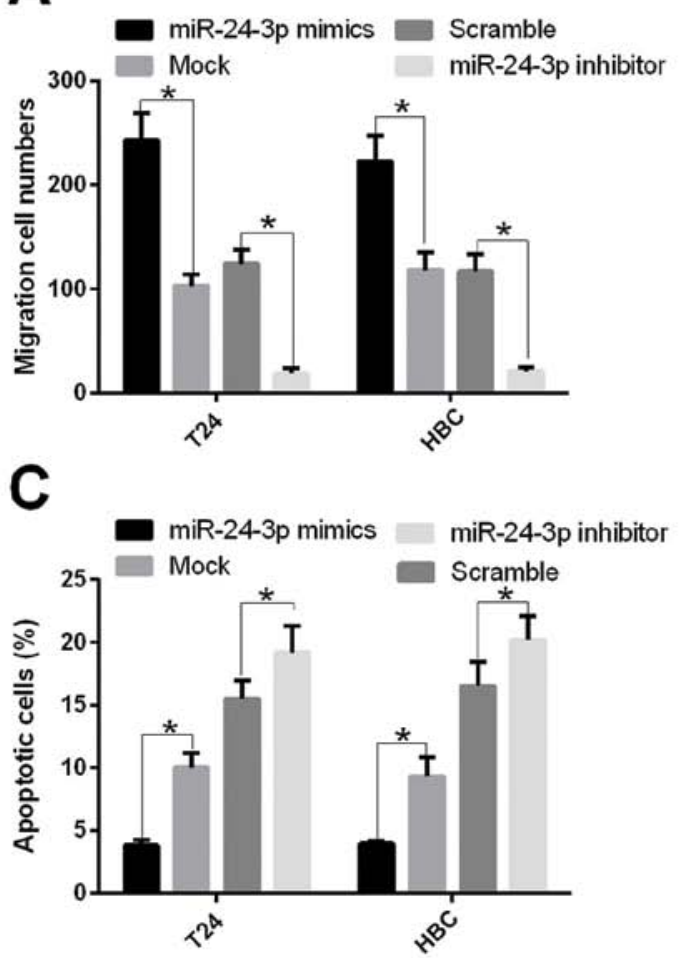

B
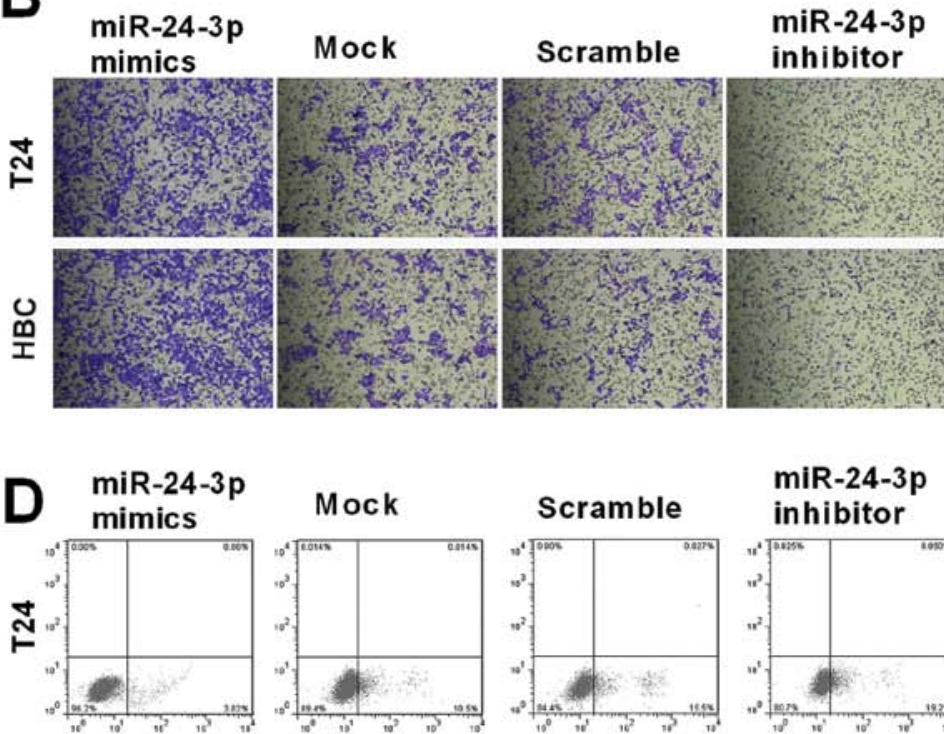

Scramble

miR-24-3p

inhibitor
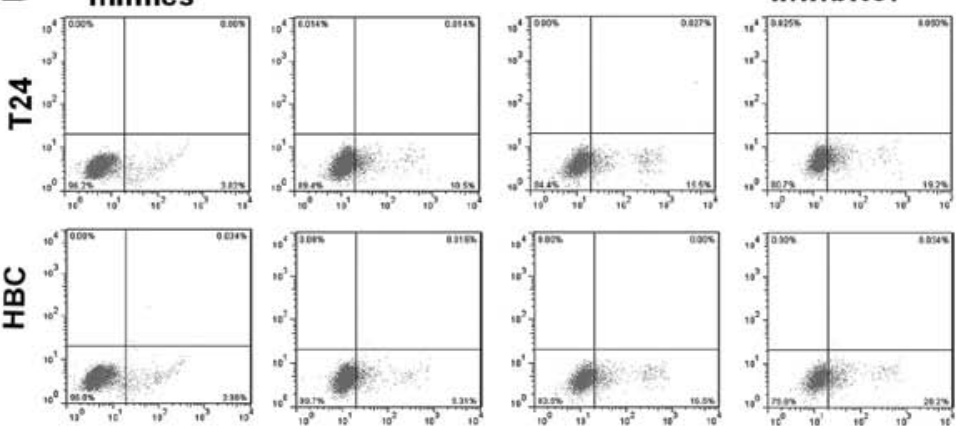
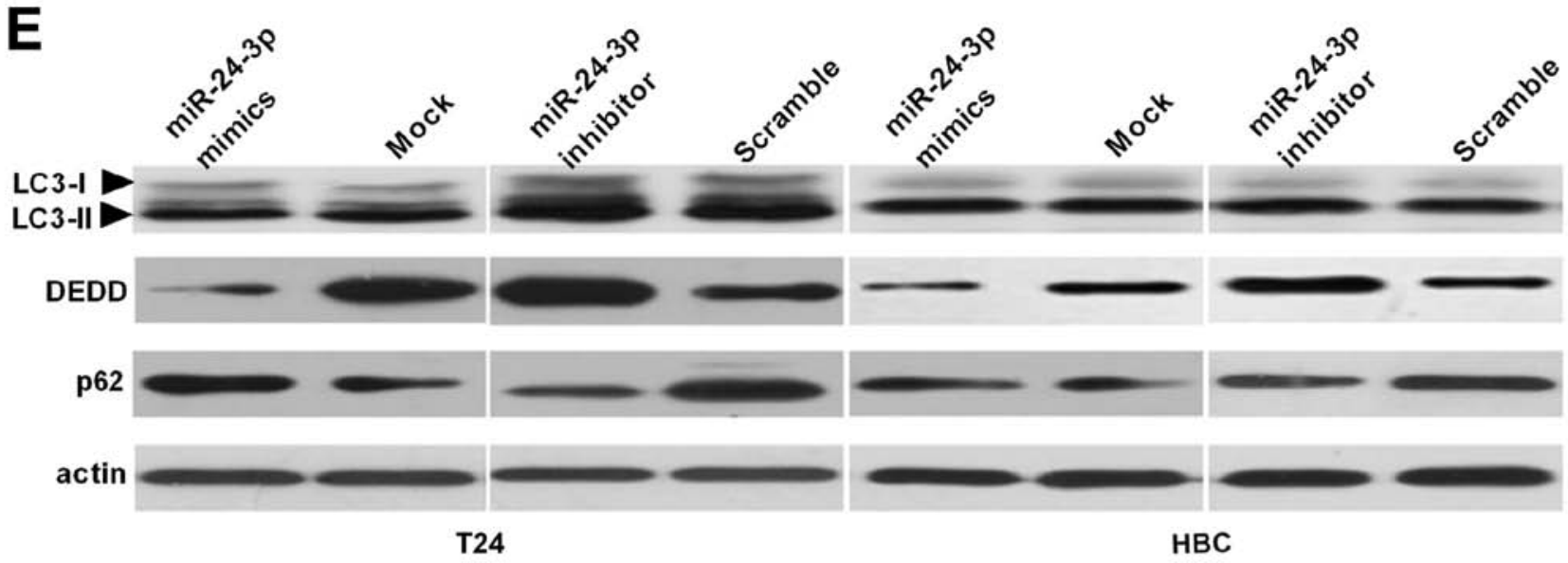

Figure 3. miR-24-3p promotes cell migration, inhibits apoptosis, and participates in autophagy by LC3, DEDD, and p62 in bladder cancer cells. (A) The migration cell numbers were counted ( $\mathrm{P}<0.05)$. (B) Cell migration was detected by the Transwell assay in T24 and HBC cells transfected with miR-24-3p mimics, mock, inhibitor or scramble. (C) Apoptotic cells were analyzed ("P<0.05). (D) Cell apoptosis was measured by Annexin V-FITC/PI staining in T24 and HBC cells transfected with miR-24-3p mimics, mock, inhibitor or scramble. (E) Western blotting detected the protein expression levels of LC3, DEDD, and p62 in T24 and HBC cells transfected with miR-24-3p mimics, mock, inhibitor or scramble. Actin was used as a protein-loading control.

DEDD and then increasing LC3 and p62 in bladder cancer cells.

miR-24-3p suppresses DEDD gene transcription in bladder cancer. To further explore the mechanism of miR-24-3p in bladder cancer, we studied the relationship between miR-24-3p and DEDD. According to TargetScan, the miR-24-3p target sites in the sequence of DEDD were found (Fig. 4A). As shown in Fig. 4B, the target sites between miR-24-3p and the DEDD 3'UTR among human and other species were also analyzed. Therefore, we hypothesized that miR-24-3p may directly bind to DEDD. The luciferase reporter gene assay was used to measure the luciferase activity of the DEDD 3'UTR. The results showed a significant decrease in fluorescence activity after cotransfection of miR-24-3p and the wild-type DEDD 3'UTR vector $(\mathrm{P}<0.01)$, but a not significant change in the mutant DEDD 3'UTR vector (Fig. 4C). The qRT-PCR results revealed that miR-24-3p inhibited the mRNA expression level of DEDD T24 and HBC cells $(\mathrm{P}<0.05)$ (Fig. 4D).

miR-24-3p promotes the growth of bladder tumor in vivo. To assess the effect of miR-24-3p on tumorigenesis in vivo, T24 cells transfected with miR-24-3p mimics, mock, inhibitor or scramble were implanted subcutaneously into nude mice. The tumor volume was calculated, and the tumor weight was measured at $0,8,16,24,32,40$, and 48 days. The results showed that mice injected with cells transfected with miR-24-3p mimics were larger than control mice $(\mathrm{P}<0.05)$ 
A

Putative binding site

DEDD-3UTR 5 . ACAGCUACCUUACC

DDD-3UR WT 5...ACAGCUACCUUACCACUGAGCCG.

miR-24-3p 3' GACAAGGACGACUUGACUCGGU

DEDD-3'UTR mut 5'...ACAGCUACCUUACCACUGCAGGG...

C

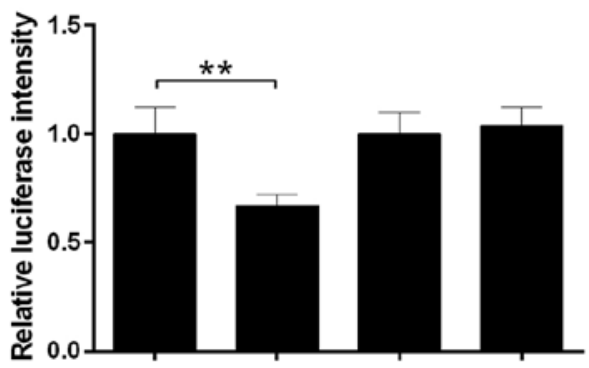

$\begin{array}{rcccc}\text { Scramble } & + & - & + & - \\ \text { miR-24-3p } & - & + & - & + \\ \text { DEDD-3'UTR-wt } & + & + & - & - \\ \text { DEDD-3'UTR-mut } & - & - & + & +\end{array}$

B

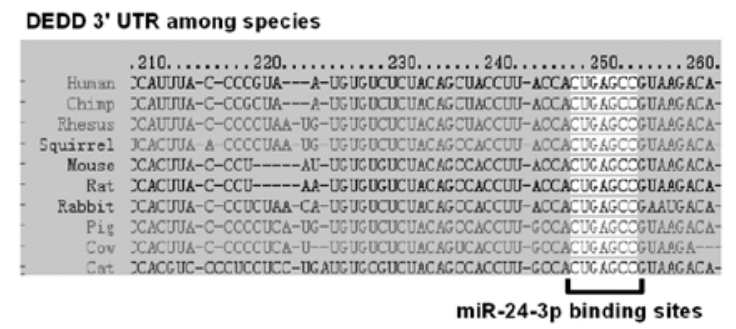

D

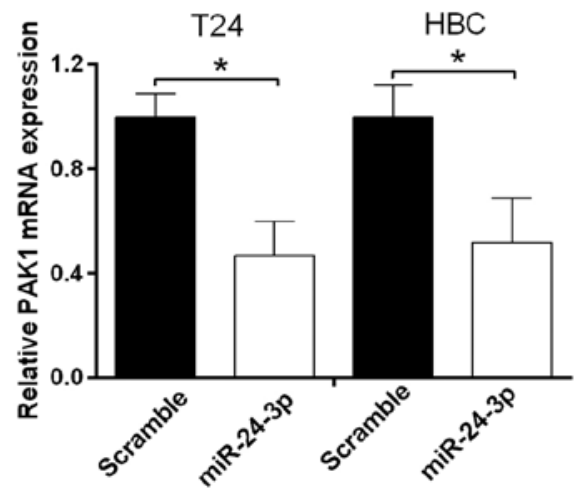

Figure 4. miR-24-3p suppresses DEDD gene transcription in bladder cancer. (A) According to the TargetScan, the miR-24-3p target site in the sequence of DEDD was predicted. (B) The target site between miR-24-3p and the DEDD 3'UTR among a variety of species was predicted. (C) The fluorescence activity of the DEDD 3'UTR was measured by the luciferase reporter gene assay in T24 cells that were cotransfected with wild-type DEDD 3'UTR and miR-24-3p or mutational type DEDD 3'UTR and miR-24-3p, respectively $\left.{ }^{(* *} \mathrm{P}<0.01\right)$. (D) qRT-PCR was used to determine the mRNA expression level of PAK1 in T24 and HBC cells transfected with miR-24-3p mimics, or scramble, respectively $\left({ }^{*} \mathrm{P}<0.05\right)$.

A

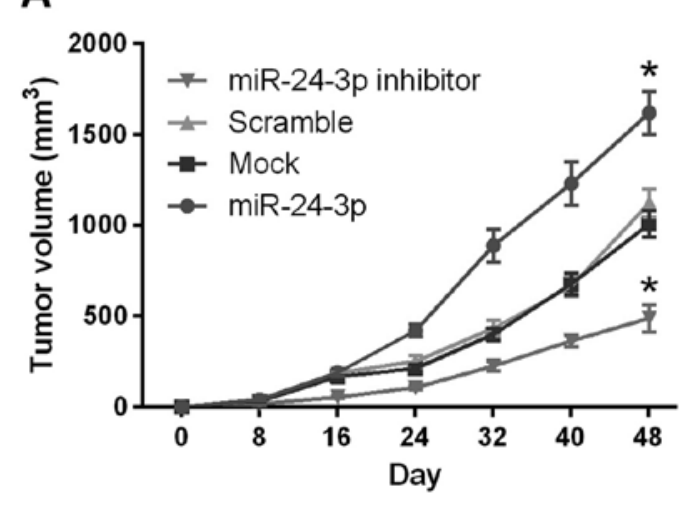

c

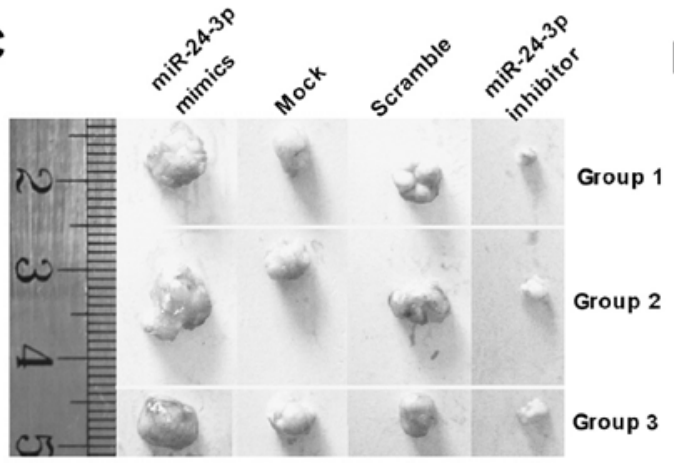

B

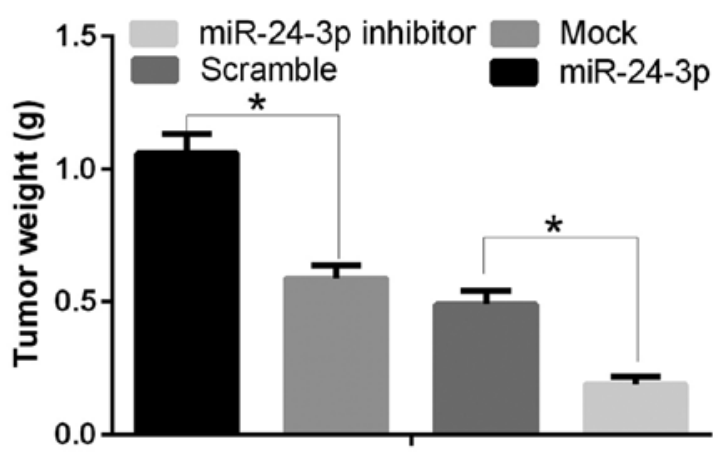

Figure 5. miR-24-3p promotes the growth of bladder tumors in vivo. (A) The tumor volume was counted in nude mice with T24 cells transfected with miR-24-3p mimics, mock, inhibitor or scramble at $0,8,16,24,32,40$, or 48 days $\left({ }^{*} \mathrm{P}<0.05\right)$. (B) The tumor weight was measured in nude mice with T24 cells transfected with miR-24-3p mimics, mock, inhibitor or scramble $\left({ }^{*} \mathrm{P}<0.05\right)$. $(\mathrm{C})$ miR-24-3p increased the tumor size in nude mice. Athymic mice were treated, and the tumors were removed and photographed. (D) The treated tumors in nude mice were removed, and RNA was extracted; the mRNA expression level of miR-24-3p was detected by qRT-PCR ( $\mathrm{P}<0.05)$. 
A
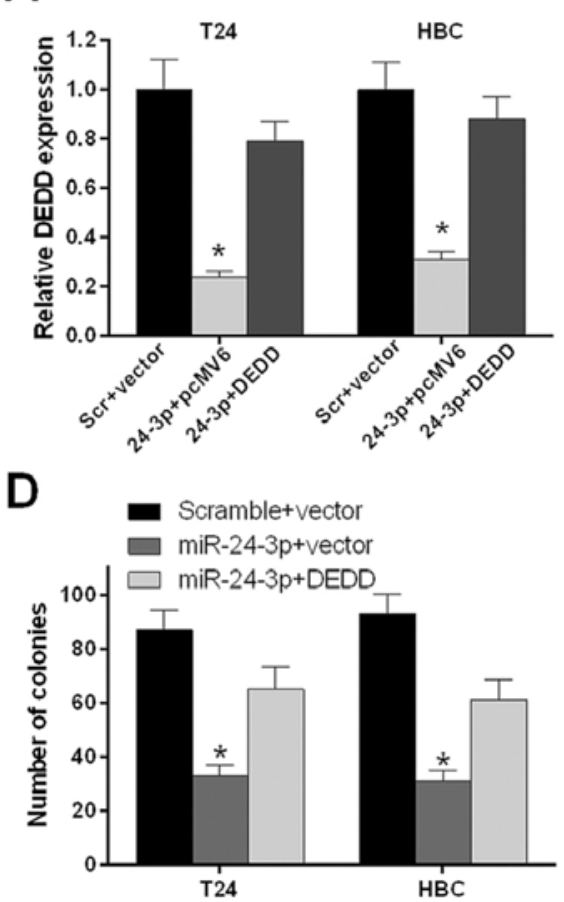

B

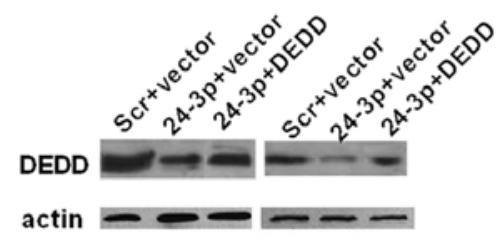

E

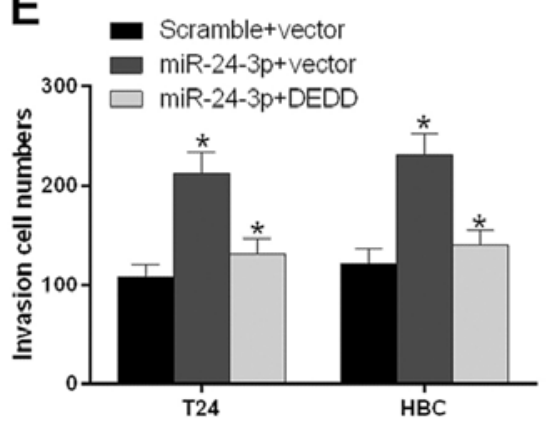

C

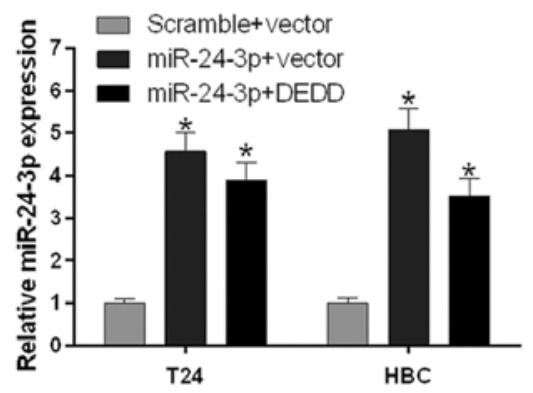

F

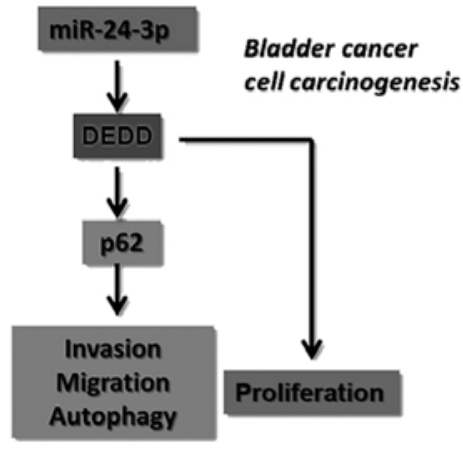

Figure 6. miR-24-3p accelerates the proliferation and invasion through DEDD in bladder cancer cells. (A) T24 and HBC cells were transfected with scramble and vector, miR-24-3p and pcMV6, miR-24-3p and DEDD, respectively. The mRNA expression level of DEDD was detected by qRT-PCR (*P<0.05). (B) The protein expression level of DEDD was detected by western blotting in T24 and HBC cells treated as in (A). Actin was used as a protein-loading control. (C) The mRNA expression level of miR-24-3p was detected by qRT-PCR in T24 and HBC cells treated as in (A) ("P<0.05). (D) Cell proliferation was detected by the colony forming assay in T24 and HBC cells treated as in A ( $\mathrm{P}<0.05$ ). (E) Cell invasion was determined by the Transwell assay in T24 and HBC cells treated as in $(\mathrm{A})\left({ }^{*} \mathrm{P}<0.05\right)$. (F) The gene network of miR-24-3p is shown in bladder cancer. miR-24-3p accelerated cell proliferation, migration, invasion and autophagy and inhibited apoptosis by inhibiting DEDD and activating p62 in bladder cancer.

(Fig. 5A). Mice injected with cells transfected with miR-24-3p mimics were heavier than control mice $(\mathrm{P}<0.05)$ (Fig. 5B). The tumors are shown in Fig. 5C, and RNA was extracted from the tumors. The qRT-PCR results indicated that the mRNA expression level of miR-24-3p was decreased significantly in mice injected with cells transfected with miR-24-3p mimics compared with mock $(\mathrm{P}<0.05)$; the mRNA expression level of miR-24-3p was increased significantly in mice injected with cells transfected with the miR-24-3p inhibitor compared with scramble $(\mathrm{P}<0.05)$ (Fig. 5D).

miR-24-3p accelerates the proliferation and invasion through DEDD in bladder cancer cells. We further investigated whether miR-24-3p expression influenced the bladder cancer cell proliferation and invasion through DEDD. T24 and HBC cells were transfected with scramble and vector, miR-24-3p and pcMV6, and miR-24-3p and DEDD, respectively. The qRT-PCR results indicated that the mRNA expression level of DEDD was decreased significantly in T24 and HBC cells transfected with miR-24-3p and pcMV6 compared with scramble and vector $(\mathrm{P}<0.05)$; the mRNA expression level of DEDD was increased significantly in T24 and HBC cells transfected with miR-24-3p and DEDD compared with those transfected with miR-24-3p and pcMV6 (P<0.05) (Fig. 6A). The western blot results also indicated that miR-24-3p inhibited the protein expression level of DEDD (Fig. 6B).

The qRT-PCR results showed that the mRNA expression level of miR-24-3p was increased significantly in T24 and
HBC cells transfected with miR-24-3p and pcMV6 compared with that in scramble and vector $(\mathrm{P}<0.05)$; the mRNA expression level of miR-24-3p was decreased significantly in T24 and HBC cells transfected with miR-24-3p and DEDD compared with miR-24-3p and pcMV6 (P<0.05) (Fig. 6C). The bladder cell proliferation ability was further detected by the colony forming unit assay. We found that miR-24-3p inhibited the proliferation ability of T24 and HBC cells and that DEDD promoted the proliferation ability of T24 and HBC cells mediated by miR-24-3p (P<0.05) (Fig. 6D). We also found that miR-24-3p promoted the invasion ability of T24 and HBC cells and that DEDD inhibited the invasion ability of T24 and HBC cells mediated by miR-24-3p ( $\mathrm{P}<0.05)$ (Fig. 6E). Therefore, as shown in Fig. 6F, miR-24-3p accelerated cell proliferation, migration, invasion and autophagy and inhibited apoptosis by inhibiting DEDD and activating p62 in bladder cancer.

\section{Discussion}

miRNAs serve as a class of small non-coding RNAs that regulate mRNAs according to the 3'UTR. Several studies have demonstrated that miRNAs are involved in multiple biological functions, including cell proliferation, migration, metastasis, and inflammation, as well as tumor angiogenesis, by targeting mRNAs in cancer $(29,30)$. Various studies have indicated that miRNAs participate in the occurrence and development of diversified diseases $(6,9,11)$. A handful of 
studies has found that various miRNAs are involved in the development of bladder cancer (31). For example, miR-126 and miR-182 are related to urinary bladder cancer (12); miR$125 \mathrm{~b}$ inhibits the development of bladder cancer through E2F3 (32); miR-143 serves as a tumor inhibitor of bladder cancer (13); miR-200 regulates EMT in bladder cancer cells and reverses the resistance to epidermal growth factor (EGF) receptor (33).

miR-24-3p has been studied in many cancers. For example, miR-24-3p promotes cell proliferation in glioma cells via MXI1 (17); the diagnosis and prognosis of miR-24-3p have been investigated in HBV-related hepatocellular carcinoma (19); miR-24-3p inhibits VP16-DDP resistance in small cell lung cancer via ATG4A(18). However, the functional relevance of miR-24-3p in bladder cancer is still not known. In our study, we indicated that the expression level of miR-24-3p was increased in bladder cancer tissues compared with paracarcinoma tissues. We also found that miR-24-3p promotes cell proliferation, migration and invasion and inhibited apoptosis in bladder cancer cells. In addition, we found that miR-24-3p promotes the growth of bladder tumors in vivo.

DEDD, an important effector molecule for cell death signaling receptors, plays critical roles in cell apoptosis, the cell cycle, and cell mitosis $(22,23)$. A recent study also reported that DEDD can weaken the EMT and inhibits tumor growth and metastasis (26). Studies have found that DEDD inhibits transforming growth factor- $\beta 1$ signaling (34). DEDD has also been correlated with apoptosis $(35,36)$ and reverses EMT by activating selective autophagy $(26,27)$. In our study, we found that miR-24-3p suppressed DEDD gene transcription. miR24-3p accelerated proliferation and invasion through DEDD in bladder cancer cells.

Autophagy, also called type 2 non-apoptotic cell death, is a prominent mechanism of self-destruction, distinguished by a catabolic process beginning with the formation of autophagosomes and degradation of cytoplasmic material in the autophagy-lysosome by lysosomal enzymes to affect health and disease (37-40). Various studies have suggested that LC3 can serve as an autophagosome marker, and p62 has been shown to be related to autophagy (41-43). Our study showed that miR-24-3p participated in autophagy through DEDD, LC3 and p62 in bladder cancer cells.

In summary, we found that miR-24-3p was highly expressed and that DEDD was expressed at a low level in bladder cancer tissues. miR-24-3p promoted proliferation, migration and invasion and inhibited apoptosis in bladder cancer cells. miR-24-3p also participated in the autophagy of bladder cancer cells through LC3, DEDD, and p62. We found that miR-24-3p promoted the growth of bladder tumors in vivo. Furthermore, miR-24-3p suppressed DEDD gene transcription. miR-24-3p accelerated proliferation and invasion through DEDD in bladder cancer cells. Therefore, our study indicated that miR-24-3p promoted bladder cancer progression by inhibiting DEDD. Thus, miR-24-3p could be a pivotal potential therapeutic target for the treatment of bladder cancer.

\section{Acknowledgements}

The study was supported by the Key Project of Education Department of Henan Science and Technology (13A320243).

\section{References}

1. Ying L, Chen Q, Wang Y, Zhou Z, Huang Y and Qiu F: Upregulated MALAT-1 contributes to bladder cancer cell migration by inducing epithelial-to-mesenchymal transition. Mol Biosyst 8: 2289-2294, 2012.

2. Bo J, Yang G, Huo K, Jiang H, Zhang L, Liu D and Huang Y: microRNA-203 suppresses bladder cancer development by repressing bcl-w expression. FEBS J 278: 786-792, 2011.

3. Egerod FL, Bartels A, Fristrup N, Borre M, Ørntoft TF, Oleksiewicz MB, Brünner N and Dyrskjøt L: High frequency of tumor cells with nuclear Egr-1 protein expression in human bladder cancer is associated with disease progression. BMC Cancer 9: 385, 2009.

4. Said N, Sanchez-Carbayo M, Smith SC and Theodorescu D: RhoGDI2 suppresses lung metastasis in mice by reducing tumor versican expression and macrophage infiltration. J Clin Invest 122: 1503-1518, 2012.

5. Iorio MV and Croce CM: MicroRNAs in cancer: Small molecules with a huge impact. J Clin Oncol 27: 5848-5856, 2009.

6. Farazi TA, Hoell JI, Morozov P and Tuschl T: MicroRNAs in human cancer. Adv Exp Med Biol 774: 1-20, 2013.

7. Djuranovic S, Nahvi A and Green R: A parsimonious model for gene regulation by miRNAs. Science 331: 550-553, 2011.

8. Kasinski AL and Slack FJ: Epigenetics and genetics. MicroRNAs en route to the clinic: Progress in validating and targeting microRNAs for cancer therapy. Nat Rev Cancer 11: 849-864, 2011.

9. Baer C, Claus R and Plass C: Genome-wide epigenetic regulation of miRNAs in cancer. Cancer Res 73: 473-477, 2013.

10. Zaman MS, Maher DM, Khan S, Jaggi M and Chauhan SC: Current status and implications of microRNAs in ovarian cancer diagnosis and therapy. J Ovarian Res 5: 44, 2012.

11. Di Leva $\mathrm{G}$ and Croce CM: The role of microRNAs in the tumorigenesis of ovarian cancer. Front Oncol 3: 153, 2013.

12. Hanke M, Hoefig K, Merz H, Feller AC, Kausch I, Jocham D, Warnecke JM and Sczakiel G: A robust methodology to study urine microRNA as tumor marker: microRNA-126 and microRNA-182 are related to urinary bladder cancer. Urol Oncol 28: 655-661, 2010.

13. Lin T, Dong W, Huang J, Pan Q, Fan X, Zhang C and Huang L: MicroRNA-143 as a tumor suppressor for bladder cancer. J Urol 181: 1372-1380, 2009.

14. Noguchi S, Yasui Y, Iwasaki J, Kumazaki M, Yamada N, Naito S and Akao Y: Replacement treatment with microRNA-143 and -145 induces synergistic inhibition of the growth of human bladder cancer cells by regulating PI3K/Akt and MAPK signaling pathways. Cancer Lett 328: 353-361, 2013.

15. Majid S, Dar AA, Saini S, Deng G, Chang I, Greene K, Tanaka Y, Dahiya R and Yamamura S: MicroRNA-23b functions as a tumor suppressor by regulating Zeb1 in bladder cancer. PLoS One 8: e67686, 2013.

16. Matsushita R, Seki N, Chiyomaru T, Inoguchi S, Ishihara T, Goto Y, Nishikawa R, Mataki H, Tatarano S, Itesako T, et al: Tumour-suppressive microRNA-144-5p directly targets CCNE1/2 as potential prognostic markers in bladder cancer. Br J Cancer 113: 282-289, 2015.

17. Xu W, Liu M, Peng X, Zhou P, Zhou J, Xu K, Xu H and Jiang S: miR-24-3p and miR-27a-3p promote cell proliferation in glioma cells via cooperative regulation of MXI1. Int J Oncol 42: 757-766, 2013.

18. Pan B, Chen Y, Song H, Xu Y, Wang R and Chen L: Mir-24-3p downregulation contributes to VP16-DDP resistance in small-cell lung cancer by targeting ATG4A. Oncotarget 6: 317-331, 2015.

19. Meng F-L, Wang W and Jia W-D: Diagnostic and prognostic significance of serum miR-24-3p in HBV-related hepatocellular carcinoma. Med Oncol 31: 177, 2014.

20. Gao Y, Liu Y, Du L, Li J, Qu A, Zhang X, Wang L and Wang C: Down-regulation of miR-24-3p in colorectal cancer is associated with malignant behavior. Med Oncol 32: 362, 2015.

21. Lu K, Wang J, Song Y, Zhao S, Liu H, Tang D, Pan B, Zhao H and Zhang Q: miRNA-24-3p promotes cell proliferation and inhibits apoptosis in human breast cancer by targeting p27Kip1. Oncol Rep 34: 995-1002, 2015.

22. Hua F, Xue J, Lü X and Hu Z: DEDD decreases Smad3 activity, promotes tumor cell apoptosis and inhibits proliferation. Yao Xue Xue Bao 48: 680-685, 2013 (In Chinese).

23. He L, Zhong G and Zhu B: microRNA-15b expressed in lung cancer-infiltrated CD8-positive memory T cell inhibit cell apoptosis by repressing DEDD. Eur J Cancer (Suppl) S147-S147, 2013. 
24. Carstens JL, Lovisa S and Kalluri R: Microenvironmentdependent cues trigger miRNA-regulated feedback loop to facilitate the EMT/MET switch. J Clin Invest 124: 1458-1460, 2014.

25. De Craene B and Berx G: Regulatory networks defining EMT during cancer initiation and progression. Nat Rev Cancer 13: 97-110, 2013

26. Lv Q, Hua F and Hu Z-W: DEDD, a novel tumor repressor, reverses epithelial-mesenchymal transition by activating selective autophagy. Autophagy 8: 1675-1676, 2012.

27. Lv Q, Wang W, Xue J, Hua F, Mu R, Lin H, Yan J, Lv X, Chen X and $\mathrm{Hu}$ ZW: DEDD interacts with PI3KC3 to activate autophagy and attenuate epithelial-mesenchymal transition in human breast cancer. Cancer Res 72: 3238-3250, 2012.

28. Jiang L, Lai YK, Zhang J, Wang H, Lin MC, He ML and Kung HF: Targeting S100P inhibits colon cancer growth and metastasis by Lentivirus-mediated RNA interference and proteomic analysis Mol Med 17: 709-716, 2011.

29. Wang J, Paris PL, Chen J, Ngo V, Yao H, Frazier ML, Killary AM, Liu CG, Liang H, Mathy C, et al: Next generation sequencing of pancreatic cyst fluid microRNAs from low grade-benign and high grade-invasive lesions. Cancer Lett 356: 404-409, 2015.

30. Stahlhut $C$ and Slack FJ: MicroRNAs and the cancer phenotype: Profiling, signatures and clinical implications. Genome Med 5: 111,2013

31. Ichimi T, Enokida H, Okuno Y, Kunimoto R, Chiyomaru T, Kawamoto K, Kawahara K, Toki K, Kawakami K, Nishiyama K, et al: Identification of novel microRNA targets based on microRNA signatures in bladder cancer. Int J Cancer 125: 345-352, 2009.

32. Huang L, Luo J, Cai Q, Pan Q, Zeng H, Guo Z, Dong W, Huang J and Lin T: MicroRNA-125b suppresses the development of bladder cancer by targeting E2F3. Int J Cancer 128: 1758-1769, 2011.

33. Adam L, Zhong M, Choi W, Qi W, Nicoloso M, Arora A, Calin G, Wang H, Siefker-Radtke A, McConkey D, et al: miR-200 expression regulates epithelial-to-mesenchymal transition in bladder cancer cells and reverses resistance to epidermal growth factor receptor therapy. Clin Cancer Res 15: 5060-5072, 2009.
34. Xue J-F, Hua F, Lv Q, Lin H, Wang ZY, Yan J, Liu JW, Lv XX, Yang HZ and Hu ZW: DEDD negatively regulates transforming growth factor- $\beta 1$ signaling by interacting with Smad3. FEBS Lett 584: 3028-3034, 2010.

35. Lee JC, Schickling O, Stegh AH, Oshima RG, Dinsdale D, Cohen GM and Peter ME: DEDD regulates degradation of intermediate filaments during apoptosis. J Cell Biol 158: 1051-1066, 2002.

36. Schutte B, Henfling M and Ramaekers FC: DEDD association with cytokeratin filaments correlates with sensitivity to apoptosis. Apoptosis 11: 1561-1572, 2006.

37. Klionsky DJ: Autophagy: From phenomenology to molecular understanding in less than a decade. Nat Rev Mol Cell Biol 8: 931-937, 2007.

38. Huett A, Goel G and Xavier RJ: A systems biology viewpoint on autophagy in health and disease. Curr Opin Gastroenterol 26: 302-309, 2010.

39. Li ZY, Yang Y, Ming M and Liu B: Mitochondrial ROS generation for regulation of autophagic pathways in cancer. Biochem Biophys Res Commun 414: 5-8, 2011

40. Liu JJ, Lin M, Yu JY, Liu B and Bao JK: Targeting apoptotic and autophagic pathways for cancer therapeutics. Cancer Lett 300: 105-114, 2011.

41. Kuma A, Matsui M and Mizushima N: LC3, an autophagosome marker, can be incorporated into protein aggregates independen of autophagy: Caution in the interpretation of LC3 localization. Autophagy 3: 323-328, 2007

42. Pankiv S, Clausen TH, Lamark T, Brech A, Bruun JA, Outzen H, Øvervatn A, Bjørkøy G and Johansen T: p62/SQSTM1 binds directly to Atg8/LC3 to facilitate degradation of ubiquitinated protein aggregates by autophagy. J Biol Chem 282: 24131-24145, 2007.

43. Ichimura Y, Kumanomidou T, Sou YS, Mizushima T, Ezaki J, Ueno T, Kominami E, Yamane T, Tanaka $\mathrm{K}$ and Komatsu M: Structural basis for sorting mechanism of p62 in selective autophagy. J Biol Chem 283: 22847-22857, 2008. 